\title{
Mucocutaneous mycoses in acquired immune deficiency syndrome (AIDS) patients hospitalised in a tertiary healthcare centre in the state of Amazonas- Brazil
}

\author{
Lindomar Schaeffer ${ }^{1}$, Diego Rayan Teixeira de Sousa ${ }^{2}$, Kátia Santana Cruz ${ }^{1}$, Lucilaide \\ Oliveira Santos ${ }^{1}$, Rossicléia Monte Lins ${ }^{1}$ and João Vicente Braga de Souza ${ }^{2 \star}$ \\ ${ }^{1}$ Laboratório de Micologia, Fundação de Medicina Tropical Dr. Heitor Vieira Dourado, Universidade do Estado do \\ Amazonas, Brazil. \\ ${ }^{2}$ Laboratório de Micologia, Instituto Nacional de Pesquisas da Amazônia, Brazil.
}

Accepted 3 August, 2012

\begin{abstract}
Several studies have been performed in which the severity of human immunodeficiency virus (HIV) infection is correlated with skin manifestations. The state of Amazonas-Brazil has a high incidence of HIV infection (2008, 26/100.000 hab), and this state is located in the tropics, a climate that promotes the development of mycoses; however, few studies have been published in the literature that discuss the prevalence of superficial mucocutaneous fungal infections in acquired immune deficiency syndrome (AIDS) patients from this region. The objective of this study was to investigate clinical forms and etiologic agents of mucocutaneous mycoses in AIDS patients hospitalised in a tertiary healthcare centre in the state of Amazonas-Brazil. Specifically, the following factors were investigated: a) the frequency of patients with mucocutaneous mycoses, b) the clinical forms and c) the main etiological agents from the mucocutaneous mycoses. One hundred and seventy patients were submitted to a dermatological examination to search for predictive manifestations of mucocutaneous mycoses. Laboratory tests (micromorphological studies and culturing) were performed on the biological samples. The samples of skin scrapings, hair and nails and the oropharyngeal swabs were transported to the mycology laboratory of the FMT-HVD for micromorphological testing (slides, $40 \% \mathrm{KOH}$ and cotton blue) and culture assays. Among the 170 patients, 116 presented clinical signs. Most of the patients were male $(68 \%)$ between the ages of 31 and 40 years old $(60 \%)$. Of the 137 sites suspected of mucocutaneous mycoses, only $\mathbf{5 2}$ were positive according to the laboratory tests. The most important clinical forms of mucocutaneous mycosis were oral candidiasis $(58 \%)$ and onychomycosis $(31 \%)$. Candida albicans and Trichophyton rubrum were the main causes of oral candidiasis and onychomycosis, respectively.
\end{abstract}

Key words: Mycoses, acquired immune deficiency syndrome (AIDS), Amazonas-Brazil.

\section{INTRODUCTION}

Regarding the issue of mucocutaneous mycoses, in a review, Alix (2009), concludes that a) superficial fungal infections represent an important cause of morbidity for people worldwide, particularly in the tropics; b) fungal

${ }^{\star}$ Corresponding

author.

joaovicentebragasouza@yahoo.com.br. infections affect the skin, hair, or the nails and tend to thrive in the heat and humidity of tropical countries and c) the environmental and cultural factors unique to the tropics, the presentations of these infections may differ from those in industrialised countries. The association of these specific situations with the incidence of acquired immune deficiency syndrome (AIDS) in the tropics creates a situation that needs investigation.

A very recent study performed by Ramos and Silva 
(2010) investigated the presence of mucocutaneous mycoses in AIDS patients from Brazil. They concluded among these patients there is a high frequency of mucocutaneous mycoses and more pronounced clinical forms. The immunological change that promotes this situation is the decrease in the number of T-helper lymphocytes, which results in a reduced capacity of macrophages to eliminate intracellular microorganisms. The main microorganisms that cause these infections are Candida spp., dermatophytes and Malassezia spp.

The relationship between the concentration of CD4 cells and skin manifestations has been evaluated in several studies. A study by Edith, Chukwuka and Anisuika (2007) demonstrated that in comparison with the CD4 cell count of asymptomatic HIV-positive patients (mean 433.6 cells $/ \mathrm{mm}^{3}$ ), the presence of various mucocutaneous manifestations in HIV-positive patients correlated with a low CD4 cell count (mean 293.63 cells $\left./ \mathrm{mm}^{3}\right) \quad(z=4.0731$ and $P<0.05)$. Such studies attempted to elucidate the pathogenesis of the lesions and to use these lesions as an indicator of the status of the immune system. In the Amazon, few studies have analysed these correlations, and even fewer studies provided descriptions of the characteristics of the mucocutaneous infections.

The objective of this study was to investigate the clinical forms and etiologic agents of mucocutaneous mycoses in AIDS patients hospitalised in a tertiary healthcare centre in the state of Amazonas-Brazil. Specifically, the following factors were investigated: a) the frequency of patients with mucocutaneous mycoses, b) the clinical forms and c) the main etiological agents of the mucocutaneous mycoses.

\section{MATERIALS AND METHODS}

\section{Model study}

A prospective cross-sectional study was conducted that investigated the clinical forms and etiologic agents of mucocutaneous mycoses in AIDS patients hospitalised in a tertiary healthcare centre in the state of Amazonas.

\section{Study characteristics}

\section{Location}

The project was developed at the Tropical Medicine Foundation Dr. Heitor Vieira Dourado (FMT-HVD), a reference hospital for infectious and parasitic diseases in the state of Amazonas-Brazil.

\section{Target Population}

Patients diagnosed with AIDS that were admitted to the FMT-HVD from July, 2006 to June, 2007.

\section{Sample}

Assuming that a) the number of patients with AIDS admitted to the FMT-HVD was approximately 360/year and b) the frequency of dermatological problems is $70 \%$ and using a confidence level of $95 \%$ and an accuracy of $5 \%$, it was determined that 170 patients should be analysed, according to the sample size calculation. The patients were included in the study by a random draw.

\section{Procedures}

\section{Frequency of patients with mucocutaneous mycoses}

The patients were submitted to a dermatological examination to search for predictive manifestations of mucocutaneous mycoses. Laboratory tests (micromorphological studies and culturing) were performed on the biological samples (skin scrapings, hair, nails and oropharyngeal swabs), which were transported to the mycology laboratory of the FMT-HVD for micromorphological testing (slides, $40 \% \mathrm{KOH}$ and cotton blue).

\section{Classification of the mucocutaneous mycoses}

In this study, the clinical forms observed were classified as oral candidosis, tinea versicolor, tinea capitis, tinea corporis, tinea cruris, tinea pedis and tinea unguium (Lacaz et al., 1998).

\section{Definition of the etiological agents}

The culture assays were performed using the Sabouraud culture medium supplemented with chloramphenicol, Sabouraud supplemented with cycloheximide and Brain Heart Infusion. The cultured filamentous fungi were identified by micromorphological assays, and the cultured yeast were identified using CHROMagar ${ }^{\mathrm{TM}}$ BD culturing, sugar assimilation and micromorphology on cornmeal agar (Lacaz et al., 1998).

\section{RESULTS}

The dermatological examination revealed that among the 170 patients, 116 had clinical signs of mucocutaneous mycoses. Twenty patients had more than one region for which there was a clinical suspicion of mucocutaneous mycosis, with a total of 137 sites suspected of mucocutaneous mycoses. The patients with clinical signs were predominantly male (68\%) and between 31 and 40 years old $(60 \%)$. Their average of CD4+ lymphocyte count was $140 \mathrm{cell} / \mathrm{mm}^{3}$, and their viral load was 59.100 copies $/ \mathrm{mL}$. From the 137 sites of suspected mucocutaneous mycoses, only 52 were confirmed by laboratory tests (Table 1). In this study, a perfect agreement was observed between the findings of the examinations by direct microscopy and the findings from the culture media.

Table 1 presents the frequency of sites of suspected mucocutaneous mycoses identified during the dermatological examination and also displays the frequency of suspected sites confirmed by laboratory mycological examination. The most important clinical forms of mucocutaneous mycosis were oral candidiasis, 
Table 1. Frequency of the clinical forms of mucocutaneous mycoses identified during the dermatological examination and the frequency of suspected sites confirmed by laboratory mycological examination.

\begin{tabular}{lcc}
\hline \multirow{2}{*}{ Clinical forms } & \multicolumn{3}{c}{ Positive results } \\
\cline { 2 - 3 } & Dermatological examination & Laboratory results \\
\hline Oral candidiasis & 68 & 30 \\
Onychomycosis & 54 & 16 \\
Tinea pedis & 5 & 0 \\
Tinea corporis & 3 & 2 \\
Tinea versicolor & 2 & 1 \\
Intertriginous candidiasis & 2 & 1 \\
Tinea cruris & 2 & 2 \\
Balanitis & 1 & 0 \\
\hline
\end{tabular}

Table 2. Frequency of the causative agents of mucocutaneous mycoses.

\begin{tabular}{llc}
\hline Clinical form & Agent & Total \\
\hline \multirow{3}{*}{ Oral candidiasis } & Candida albicans & 21 \\
& Candida tropicalis & 3 \\
& Candida parapsilosis & 3 \\
& Candida albicans + Candida tropicalis & 3 \\
& Trichophyton rubrum & 8 \\
Onychomycosis & Candida albicans & 3 \\
& Candida tropicalis & 3 \\
Tinea corporis & Candida parapsilosis & 2 \\
Tinea versicolor & & \\
Intertriginous candidiasis & Trichophyton rubrum & 2 \\
Tinea cruris & Malassezia spp. & 1 \\
\hline
\end{tabular}

onychomycosis and tinea pedis.

Table 2 presents the frequency of the causative agents of the mucocutaneous mycoses. Candida albicans and Trichophyton rubrum were the main causes of oral candidiasis and onychomycosis, respectively.

\section{DISCUSSION}

In the present work, 116 of the $170(68 \%)$ patients that were submitted to dermatological examination presented clinical signs. This high frequency of dermatological alterations was expected because previous studies performed by Blanes et al. (2010) demonstrated that the prevalence of dermatoses in HIV/AIDS patients is approximately $98.3 \%$. The most common conditions are xerosis, seborrheic dermatitis, distal subungual onychomycosis and viral warts. The age and sex distributions of the patients were similar to those described in previous works (Brilhante et al., 2000; Blanes et al., 2010). Only 52 of the 137 sites that were investigated for mucocutaneous fungal infection were confirmed to in infected by laboratorial examinations; this discrepancy may be attributable to the following variables: a) the clinical form of the mycoses; b) the number of patients previously treated; c) the experience of the dermatologists; d) the level of training of the microscopists; e) the laboratory conditions and f) the species of microorganisms that are most prevalent (Brilhante et al., 2000).

Regarding the clinical forms of the mucocutaneous fungal infection, candidiasis (58\%), onychomycosis (31\%) and tinea pedis (4\%) were the most frequent. In a recent study performed by Monsel et al., (2008), with a similar population, a high frequency of candidiasis (53\%) was also observed. The high frequency of onychomycosis observed in the present work was also observed in a study carried out by Cambuim et al., (2011), in Brazilian hospitals (32\%). The results of a series of previous studies agree with the results of this work and demonstrated that these two clinical forms (candidiasis and onychomycosis) are closely linked with HIV/AIDS. The distribution of mucocutaneous diseases in patients without evident immunodeficiency is different. Kamothi et 
al., (2010), demonstrated high frequencies of tinea corporis $(41 \%)$ and tinea cruris (21\%), and Neela (2007), observed high frequencies of tinea corporis (63\%) and tinea pedis (15\%).

Regarding the species of fungi responsible for the mucocutaneous mycoses in the present study, $C$. albicans and $T$. rubrum were the main causative agents of oral candidiasis and onychomycosis, respectively. This result for oral candidiasis is consistent with the results published in the literature, which have been demonstrated that $C$. albicans, $C$. tropicalis and $C$. glabrata are the most important causative agents (Monsel et al., 2008). One fact that should attract attention is the existence of three cases of oral candidiasis caused by $C$. albicans and $C$. tropicalis simultaneously; future studies should be conducted to investigate these findings, which were first reported after the utilisation of chromogenic culture media such as CHROMagar TM BD. In the case of onychomycosis, the results are similar those obtained by Graham et al., (2008), showing that the main causative agent was $T$. rubrum; however, in the present study observed a higher involvement of Candida spp. was involved. In addition, in the work of Graham et al., (2008), a higher involvement of dermatophytes ( $T$. mentagrophytes, $T$. tonsurans and Epidermophyton floccosum) was observed.

The present manuscript agree with Ramos and Silva (2010) founds demonstrating that among Aids patients there is a high frequency of mucocutaneous mycoses and more pronounced clinical forms. In the investigation conditions, it was not found fungal infections caused by atypical fungi; it was observed only similar species previous described in investigations with AIDS patients.

\section{ACKNOWLEDGEMENTS}

The authors are grateful to the Ministério da Ciência e Tecnologia (MCT-INPA) and FAPEAM for financial support.

\section{REFERENCES}

Alix JC (2009). Superficial cutaneous fungal infections in tropical countries. Dermatol. Ther. 22:550-559.

Blanes M, Belinchón I, Merino E, Portilla J, Sánchez-Payá J, Betlloch I (2010). Current Prevalence and Characteristics of Dermatoses Associated with Human Immunodeficiency Virus Infection Actas Dermosifiliogr. 101(8):702-709.

Brilhante RSN, Paixão GC, Salvino LK, Diógenes MJN, Bandeira SP, Rocha MFG, Santos JBF, Sidrin JJC (2000). Epidemiology and ecology of dermatophytosis in the city of Fortaleza: The Trichophyton tonsurans as important emergent patogen of the Tinea capitis. Fortaleza, Brasil. Rev. Soc. Bras. Med. Trop. Set/Out 33(5):417-425.

Cambuim IIFN, Macêdo DPC, Delgado M, Lima KM, Mendes GP, Souza-Motta CM, Lima DMM, Fernandes MJ, Magalhães OMC, Queiroz LA e Neves RP (2011). Avaliação clínica e micológica de onicomicose em pacientes brasileiros com HIV/AIDS Revista da Sociedade Brasileira de Medicina Tropical 44(1):40-42, Jan-Fev.

Edith NN, Chukwuka JC, Anisuiba B (2007). Correlation of mucocutaneous manifestations of HIV/AIDS infection with CD4 counts and disease progression. Int. J. Dermatol. 46(Suppl. 2):14-18.

Graham ERJ, Charlene LB, Loan T, Raza A (2008). The prevalence of dermatophyte infection in patients infected with human immunodeficiency virus. Int. J. Dermatol. 47:339-343.

Kamothi MN, Patel BP, Mehta SJ, Kikani KM, Pandya JM (2010). Prevalence of Dermatophyte Infection In: District Rajkot Electronic J. Pharmacol. Ther. 3:1-3.

Lacaz CS, Porto E, Heins-Vaccari EM, Melo NT (1998). Dermatophytes. In: Guide for identification of Fungus, Actinomicets and Algae of medical interest. Sarvier. S. Paulo, Brasil. pp. 55-85.

Monsel GF, Ly ACP, Diousse B, Ndiaye EC (2008). Prevalence of skin disorders in HIV patients in Senegal and relationship to degree of immunosuppression Annales de dermatologie et de vénéréologie, 135:187-193.

Ramos-e-Silva M, Lima CMO, Schechtman RC, Trope BM, Carneiro S (2010). Superficial mycoses in immunodepressed patients (AIDS). Clin. Dermatol. 28:217-225. 